\title{
Knowledge, Attitudes and Practices of Healthcare Professionals towards Antimicrobial Stewardship and Their Predictors in Fitche Hospital \\ Gobazie Temesgen Tegagn ${ }^{1}$, Tadele Mekuriya Yadesa ${ }^{{ }^{*}}$ and Yasin Ahmed ${ }^{2}$
}

${ }^{1}$ Department of Pharmacy, College of Medicine and Health Science, Ambo University, Ambo, Ethiopia

${ }^{2}$ Pharmacist at Ministry of Health, Ethiopia

\begin{abstract}
Background: The recognized effects of inappropriate use of antibiotics are multiple: rapid emergence of resistance, selection pressure on resistant microorganisms, adverse reactions, and treatment failures. An effective approach to improving antimicrobial use in hospitals is an organized antimicrobial management program known as Antimicrobial Stewardship (AMS). It is used by healthcare institutions to reduce inappropriate antimicrobial use, improve patient outcomes and reduce adverse consequences of antimicrobial use.
\end{abstract}

Objective: The aim of this study is to assess knowledge, attitudes and practices of healthcare professionals towards antimicrobial stewardship and their predictors in Fitche hospital.

Methods: A prospective cross-sectional study was designed based on an endorsed anonymous self-administered questionnaire. Stratified random sampling with proportional allocation was applied for sampling. The inclusion criteria for this study are all healthcare professionals who are willing to participate in the study excluding laboratory technicians and radiologists. Data was collected from April 20 to May 24, 2016, compiled, analyzed (using descriptive statistics and binary logistic regression) and presented using frequency table, figures and charts.

Results: There were a total of 107 participants. $64.5 \%$ of the respondents were males. More than half of participant had good knowledge $(68.2 \%), 16 \%$ had positive attitude towards antimicrobial stewardship and $78 \%$ of Health Care Professionals had good practices related to antimicrobial stewardship. Age $(p=0.354 S D=0.4)$, profession $(p=0.52)$ and years of experience $(p=0.125)$ are not significant predictors of health care professionals knowledge, attitude and practices towards Antimicrobial Stewardship.

Conclusion and recommendation: Despite the fact that the practice of Antimicrobial Stewardship is not implemented and well developed in most of hospitals and healthcare facilities, the level of Knowledge Attitude Practice towards the Antimicrobial Stewardship was good. However, surprisingly, in spite of the casual knowledge and selfreported practices of our study participant's, attitude with regards to antibiotic use were found to be unsatisfactory.

Keywords: Antimicrobial stewardship; Resistance; Knowledge; Attitude; Practice

\begin{abstract}
Abbreviations: ASP: Antibiotics Stewardship Program; CSA: Central Statistical Agency of Ethiopia; AMR: Antimicrobial Resistance; SMART: Study for Monitoring Antimicrobial Resistance Trend; FGH: Fitche General Hospital; MDRO's: Multi Drug Resistant Organisms; HCP: Healthcare Professionals; AS: Antibiotics Stewardship; TB: Tuberculosis; DM: Diabetes Mellitus; HIV: Human Immune Deficiency Virus; AIDS: Acquired Immune Deficiency Syndrome; WHO: World Health Organization; ICU: Intensive Care Units; KAP: Knowledge, Attitude and Practice
\end{abstract}

\section{Introduction}

Antibiotics are among the most commonly used and misused of all drugs. The inevitable consequence of the widespread use of antimicrobial agents resulted in the emergence of antibiotic-resistant pathogens, fueling an ever-increasing need for new drugs. However, the pace of antimicrobial drug development has slowed dramatically, with only a handful of new agents, few of which are novel, being introduced into clinical practice each year [1-5].

Reducing inappropriate antibiotic use is thought to be the best way to control its negative consequences. Although awareness of the consequences of antibiotic misuse is increasing, overprescribing remains widespread, driven largely by patient demand, time pressure on clinicians, and diagnostic uncertainty [6-9]. Optimal and judicious selection of antibiotics for infectious disease therapy requires clinical judgment and detailed knowledge of pharmacological and microbial factor $[10]$

Antimicrobial stewardship has been defined as "the optimal selection, dosage, and duration of antimicrobial treatment that results in the best clinical outcome for the treatment or prevention of infection, with minimal toxicity to the patient and minimal impact on subsequent resistance [11]". It involves appropriate selection, dosing, route of administration, and duration of antimicrobial therapy (i.e., the prudent use of antibiotics) [11]. Use of antimicrobial stewardship in combination with infection prevention and control efforts limits the emergence and transmission of antimicrobial-resistant pathogens [11-13].

The primary goal of antimicrobial stewardship is to optimize

*Corresponding authors: Tadele Mekuriya Yadesa, Lecturer and Clinical Pharmacy Specialist, Department of Pharmacy, College of Medicine and Health Science, Ambo University, Ambo, Ethiopia, Tel: +251471110331; E-mail: maatiikoo4@gmail.com

Received February 26, 2017; Accepted March 15, 2017; Published March 22 , 2017

Citation: Tegagn GT, Yadesa TM, Ahmed Y (2017) Knowledge, Attitudes and Practices of Healthcare Professionals towards Antimicrobial Stewardship and Their Predictors in Fitche Hospital. J Bioanal Biomed 9: 091-097. doi:10.4172/1948 593X.1000159

Copyright: @ 2017 Tegagn GT, et al. This is an open-access article distributed under the terms of the Creative Commons Attribution License, which permits unrestricted use, distribution, and reproduction in any medium, provided the original author and source are credited. 
clinical outcomes while minimizing the unintended consequences of antimicrobial use. Reducing health care costs without adversely affecting the quality of care is a secondary goal of antimicrobial stewardship [11].

Antimicrobial Resistance (AMR) has emerged as a major public health problem in different countries as evidenced by several studies. Ethiopian hospitals consume about $50 \%$ of the national drug budget, which are considered to have high drug budget compared to the population segment using these health facilities. However, very little is known how drugs particularly antibiotics are used in hospitals like in other health facilities [14-17].

The aim of this study is to assess knowledge, attitudes and practices of healthcare professionals towards antimicrobial stewardship and their predictors. Owing to the lack of similar studies in the area, the results from this study can be utilized in benefiting patients by identifying problems associated with inappropriate use of antibiotics. Over all the study result will help in developing scheme in reducing health care costs related to antimicrobial resistance without adversely affecting the quality of care.

\section{Methods and Participants}

\section{Study area and study period}

The study was conducted in Fitche general hospital and Fitche health center from April 20 to May 242016 in Fitche town. Fitche is located in Oromia regional state, north shewa zone at a distance of $115 \mathrm{~km}$ from Addis Ababa. The hospital has different departments and wards like Outpatient Department (OPD), medical ward, gynecology and obstetrics ward, pediatrics ward and surgical ward. It delivers diversified health services and clinics including the emergency services, eye clinic, dental clinic, mother and child health $(\mathrm{MCH})$, psychiatry clinic, laboratory, X-ray, physiotherapy and follow up of chronic disease like TB, and HIV AIDS. The hospital possesses outpatient, inpatient, emergency and ART pharmacies.

\section{Study design}

Prospective cross sectional study was conducted via anonymous self-administered questionnaire to healthcare providers within Fitche town.

\section{Populations}

Source population: All health care professionals in Fitche town.

Study population: The study populations are all healthcare providers who fulfilled the inclusion criteria and attending at Fitche general hospital and health center in Fitche town during study period.

Inclusion criteria: All healthcare professionals, who are willing to participate in the study.

Exclusion criteria: Laboratory technicians and radiologists.

\section{Sample size and sampling technique}

Sample size determination: Sample size is calculated from the total study population that fulfill in inclusion criteria by the following formula:

$$
\mathrm{n}=\mathrm{Z}^{2} \mathrm{P}(1-\mathrm{P}) / \mathrm{D}^{2}
$$

Where:

- n-Sample size;

- Z-Confidence level=95\% (1.96);
- P-Anticipated proportion $=50 \%$ (0.5) to allow maximum sample size;

- D-Margin of errors=5\% (0.05).

There were 140 healthcare professionals who fulfill inclusion criteria.

$\mathrm{N}=140$

Therefore the corrected sample size is calculated as:

$\mathrm{No}=\mathrm{n} /(1+\mathrm{n} / \mathrm{N})$

$\mathrm{Nf}=384 /(1+384 / 140)=102$

Allowance of $5 \%=0.05 \times 10^{5}=5$

Therefore total sample size $=107$

Proportional sampling technique is used. The proportion of the candidates of the study from their respective Profession is calculated as follows:

- Physician=13, then the sample will be taken 107/140(13)=10;

- Pharmacists \& druggists=09, then the sample will be taken 107/140(9)=7;

- Nurse in all type $=82$, then $107 / 140(82)=63$;

- Midwives=15 then $107 / 140(15)=11$;

- Health officer $=6$ then $107 / 140(6)=5$;

- Health extension worker $=14$ then $107 / 140(14)=11$; Total $=107$.

Sampling technique: Stratified random sampling was used and proportional allocation to each stratum.

\section{Study variables}

- Independent variables: Age, Sex, Professional/occupation, and Year of experience.

- Dependent variables: Knowledge, Attitude, and Practice.

\section{Data collection tool and procedure}

A questionnaire with 3 parts containing questions about knowledge (knowledge about AMR, familiarity with terms and knowledge on effectiveness of ASP) attitude (overall attitude about antimicrobial resistance) and practices (related to prescribing) was distributed among healthcare professionals who fulfill the inclusion criteria and are willing to participate in the study. The questionnaire also consisted of a list of possible causes which could be responsible for the development of antibiotic resistance and HCP were asked to rate them according to their importance.

\section{Data quality assurance}

The clarity and completeness checkup of data collection formats was carried out before the actual data collection and data clearing was done every day; questionnaire with incomplete information was excluded from the study to avoid error. Then collected data was processed and analyzed cautiously in the line of its objective.

\section{Data processing and analysis}

Once all necessary data was obtained and checked for completeness, sorted and categorized accordingly. Then the data was entered and analyzed using the Statistical Package for Social Sciences (SPSS ${ }^{\circledR} 20.0$, 
Citation: Tegagn GT, Yadesa TM, Ahmed Y (2017) Knowledge, Attitudes and Practices of Healthcare Professionals towards Antimicrobial Stewardship and Their Predictors in Fitche Hospital. J Bioanal Biomed 9: 091-097. doi:10.4172/1948-593X.1000159

USA) and interpreted. Then the final result was compared with the standard criteria and was presented using tables, diagrams and various graphs.

\section{Ethical consideration}

A Formal letter was obtained from Ambo university Department of pharmacy in order to get permission to conduct the study. Informed consent was obtained from each participant in each questionnaire after the purpose of the study was explained to respondent. Confidentiality of the information was assured and privacy of the respondent was maintained.

\section{Operational definitions}

Knowledge is accordingly the concepts and information that HCPs have regarding to ASP.

Good knowledge: When the respondents agree on $\geq 65 \%$ of the statement of knowledge.

Poor knowledge: When the respondents agree on $<65 \%$ of the statement of knowledge.

Attitude is the perception and internal feeling that HCPs possess towards ASP which may be positive or negative.

Positive attitude: When the respondents agree on $>75 \%$ of the statements of attitude.

Negative attitude: When the respondents agree on $<75 \%$ of the statements of attitude.

Practice is the activities of HCPs towards ASP.

- Good practice-when the respondents agree on $>70 \%$ of the statement of practice.

- Poor practice-when the respondents agree on $<70 \%$ of the respondent of practice.

Healthcare professionals (HCPs) in this regards it mean any healthcare providers. $\sqrt{ }$ From the 7 selected questions that assess attitude, if the participants agreed with $75 \%$ or more statements, she/he said to be had positive attitude.

$\sqrt{ }$ From the 7 selected questions that assess practice, if the respondent is correctly answer $70 \%$, she/he said to be had good practice.

\section{Results}

\section{Socio demographic characteristics of study participants}

Of the total of 107 study participants, over half (64.5\%) of them were males, $89(83.2 \%)$ belonged to age group of $20-30$ years and 63 (58.9\%) of them were nurses (Table 1 ).

\section{Knowledge of healthcare professionals on ASP}

Majority of HCP (49.5 agreed and 35.5 of HCP strongly agreed) on if antibiotics are used inappropriately they can lead to resistance. Most of HCP (48.8\% agreed 35.5\% strongly agreed) on incorrect use of antibiotics can lead to ineffective treatment. They also agreed incongruous use of antibiotics can lead to increased adverse effects (57.9\%) and additional burden of medical cost to the patient (54.2\%) (Figure 1).

\begin{tabular}{|c|c|c|c|}
\hline & & Category & Frequency (\%) \\
\hline \multirow{2}{*}{1} & \multirow{2}{*}{ Sex } & Male & $69(64.5 \%)$ \\
\hline & & Female & $38(35.5 \%)$ \\
\hline \multirow{3}{*}{2} & \multirow{3}{*}{ Age } & $20-30$ & $89(83.2 \%)$ \\
\hline & & $31-40$ & $16(15.0 \%)$ \\
\hline & & $>40$ & $2(1.9 \%)$ \\
\hline \multirow{6}{*}{3} & \multirow{6}{*}{ Profession } & Doctor & $10(9.3 \%)$ \\
\hline & & Nurse & $63(58.9)$ \\
\hline & & $\mathrm{HO}$ & $5(4.7 \%)$ \\
\hline & & Pharmacy & $7(6.5 \%)$ \\
\hline & & Midwife & $11(10.3 \%)$ \\
\hline & & Others & $11(10.3 \%)$ \\
\hline \multirow{2}{*}{4} & \multirow{2}{*}{ Years of experience } & $0-4$ & $80(78.4 \%)$ \\
\hline & & $5-9$ & $27(25.2 \%)$ \\
\hline
\end{tabular}

Table 1: Age, sex distribution, profession and years of experience of healthcare professionals who were working in Fitche General Hospital.

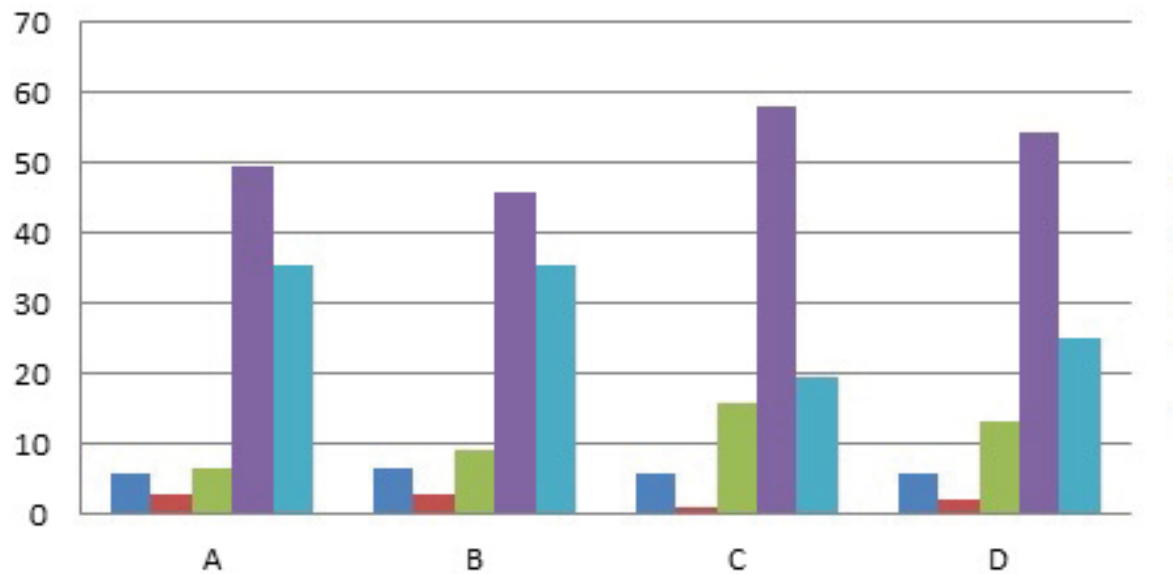

A

A. Inappropriate antibiotics use can lead to resistance.

B. Inappropriate antibiotics use can lead to ineffective treatment

C. Inappropriate antibiotics use can lead to increase adverse effects

D. Inappropriate antibiotics use gives additional burden of medical cost to the patient

Figure 1: Basic knowledge of healthcare professionals about antibiotic stewardship. 
Citation: Tegagn GT, Yadesa TM, Ahmed Y (2017) Knowledge, Attitudes and Practices of Healthcare Professionals towards Antimicrobial Stewardship and Their Predictors in Fitche Hospital. J Bioanal Biomed 9: 091-097. doi:10.4172/1948-593X.1000159

\section{Familiarity of healthcare professionals with terms}

More than half of healthcare professionals (59.8\%) were very familiar with the term antibiotics resistance whereas nearly half of healthcare professionals are not familiar with the term antimicrobial stewardship (48.6\%) and antibiogram (43\%) (Figure 2).

\section{Knowledge of healthcare professionals about ASP effectiveness with respect to the following conditions}

Half of HCP reply that ASP is effective in improving patient outcomes $(50.5 \%)$. Nearly half of them respond that, ASP is effective in improving patient safety $(45.8 \%)$ and about $(41.1 \%)$ respond ASP is effective in reducing resistance (Figure 3 ).

\section{Attitude of healthcare professionals on AS}

Attitude of healthcare professionals about antimicrobial use and resistance: Most of HCP do not believe that antibiotics are overused (43\%) and AMR is a great problem in their facility (35.5\%) and $74.8 \%$ of them disagree on appropriate use of antimicrobial can lead to resistance. Unfortunately, $31.8 \%$ of HCP believe new antimicrobial development will keep up with the current resistance needed and $57 \%$ of them were disagreed/strongly disagreed on restriction of antibiotics for controlled use (Figure 4).

\section{Practices related to ASP}

Practices when treating patients with infectious diseases: The main of source of information used by HCP in dealing with infectious disease were standard treatment guidelines $(68.2 \%)$ followed by PubMed and Up-to-date (18.7\%) (Table 2).

More than half of healthcare professionals have responded community acquired infections have higher risks of developing resistance (57.9\%) than healthcare associated (nosocomial infection) (42.1\%). Full recovery was mentioned by majority of HCP as the clinical condition for the patient to shift from parenteral to oral antibiotics (Table 3).

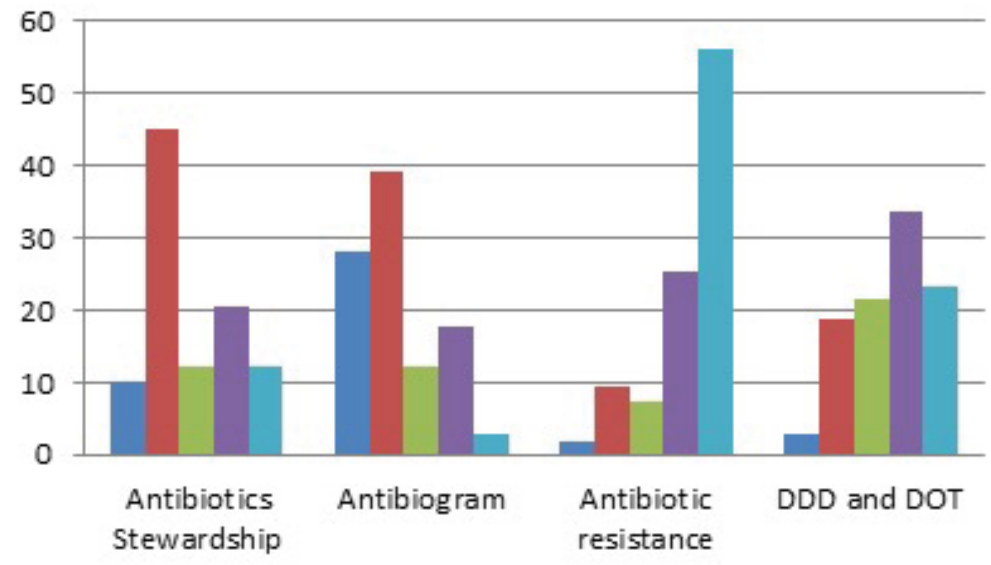

Not at all fam iliar(I've never heard of it)

Not familiar(I heard the term but I'm not sure what it is)

- Somehow familiar

nfamiliar(I've heard the term and have som e fam iliarity)

- V ery Fam iliar( ingaged in practice)

Figure 2: Familiarity of healthcare professionals with terms

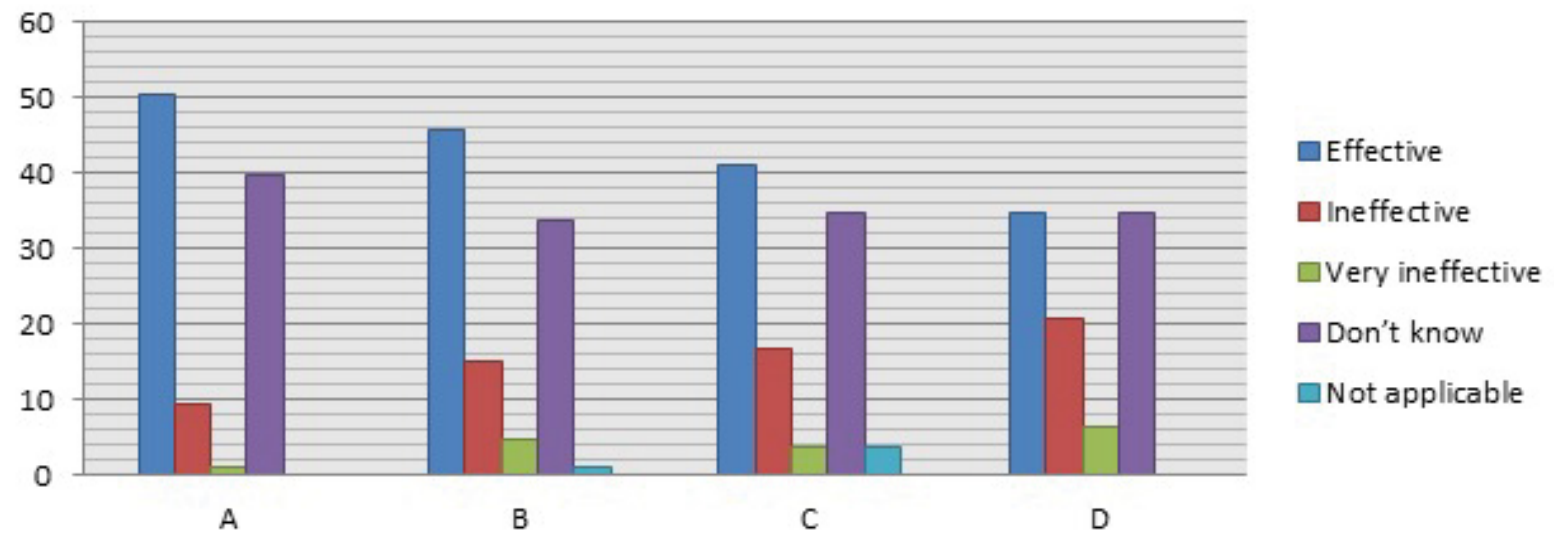

A. Improving patient outcomes.

B. Improving Patient safety (Minimize unintended consequences of antimicrobials).

C. Reducing resistance.

D. Reduce healthcare costs (without adversely impacting quality of care).

Figure 3: Knowledge of healthcare professionals on effectiveness of antibiotics steward ship. 
Citation: Tegagn GT, Yadesa TM, Ahmed Y (2017) Knowledge, Attitudes and Practices of Healthcare Professionals towards Antimicrobial Stewardship and Their Predictors in Fitche Hospital. J Bioanal Biomed 9: 091-097. doi:10.4172/1948-593X.1000159

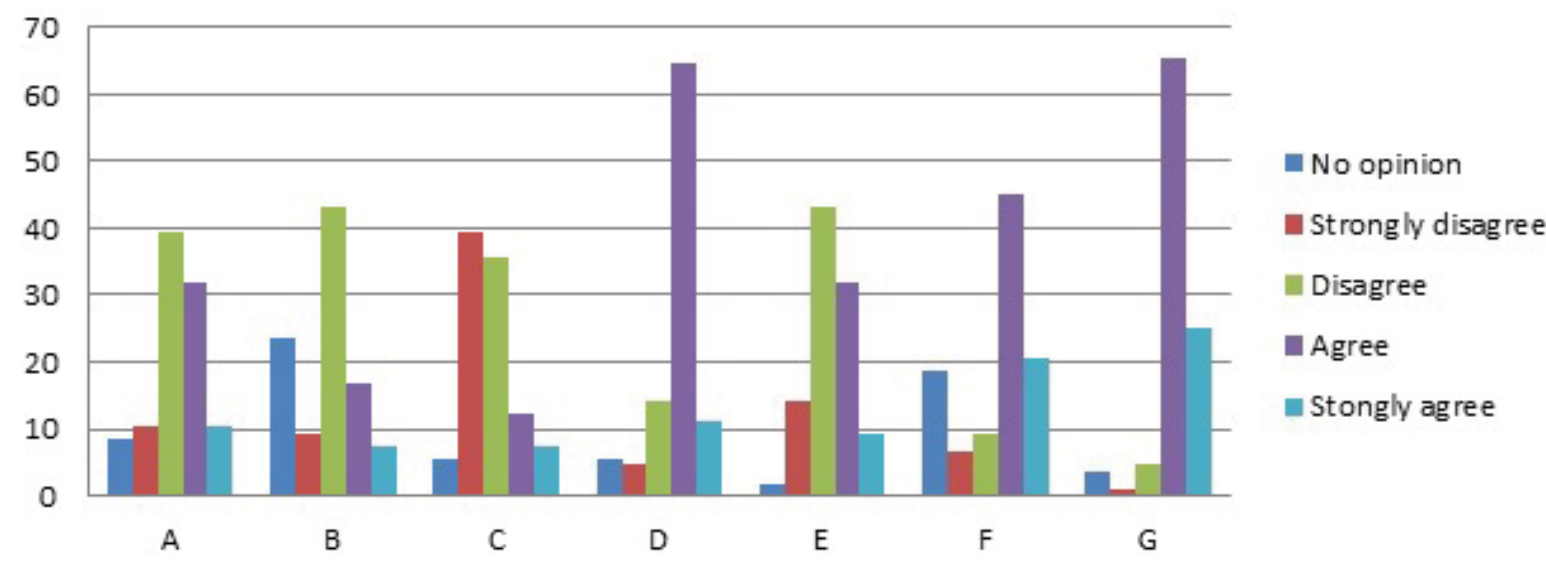

A. Antimicrobials are overused at my hospital/facility.

B. Antimicrobial resistance is a great problem in my hospital/facility.

C. Appropriate use of antimicrobials can cause antimicrobial resistance.

D. New antimicrobial development will keep up with our current resistance needs.

E. Restriction on antimicrobials use is reasonable method for controlling antibiotic use.

F. Antibiotic resistance is an important and serious public health issue facing the world.

G. I would like more education on appropriate use of antibiotics.

Figure 4: Attitude of healthcare professionals about antimicrobial use and resistance.

\begin{tabular}{|c|c|c|}
\hline & Sources & Frequency \\
\hline 1 & STG & $73(68.2 \%)$ \\
\hline 2 & PubMed and up-to-date & $20(18.7 \%)$ \\
\hline 3 & Wikipedia and medical encyclopedia & $1(0.9 \%)$ \\
\hline 4 & Ask a colleague & $1(0.9 \%)$ \\
\hline 5 & Clinical textbooks & $10(9.3 \%)$ \\
\hline 6 & Smart phones internet & $2(1.9 \%)$ \\
\hline
\end{tabular}

Table 2: Sources of information used in dealing with treatment of infectious diseases.

\begin{tabular}{|c|c|c|}
\hline & Parameters & Frequency \\
\hline 1 & Normal heart rate & $10(9.3 \%)$ \\
\hline 2 & Normal blood pressure & $12(11.2 \%)$ \\
\hline 3 & Normal respiratory rate & $1(0.9 \%)$ \\
\hline 4 & Normal body temperature & $11(10.3 \%)$ \\
\hline 5 & Full recovery & $73(68.2 \%)$ \\
\hline
\end{tabular}

Table 3: Clinical conditions used by HCP to shift from parenteral to oral antibiotics.

Practices which contributes to resistance in hospitals: Majority of healthcare professionals mention noncompliance and poor adherence to therapy as the main factor behind the emergence of resistance in hospitals followed by excessive and prolonged antimicrobial therapy (28\%). Adequate staff education regarding MDROs was needed by majority of HCP (80.3\%) (Table 4).

Antibiotic prescribing and other practices: In terms of practices related to prescribing, $48.5 \%$ agreed and $36.4 \%$ strongly agree that microbiology lab results must be provided to prescribers and $55.1 \%$ of the respondents believed restrictions on antibiotics impair the ability to provide good patient care (Figure 5).

The level of knowledge on antimicrobial stewardship was good among the majority $(62.8 \%)$ of the participants. Good practice was also observed among most (72.9\%) of the participants. However, positive

\begin{tabular}{|c|c|c|}
\hline \multicolumn{2}{|c|}{ Factors } & Frequency \\
\hline 1 & Large number of patients with close proximity to each other & $5(4.7 \%)$ \\
\hline 2 & Intensive and prolonged antimicrobial therapy & $30(28.0 \%)$ \\
\hline 3 & $\begin{array}{c}\text { Treatment with contaminated medical equipment and } \\
\text { hands of staff }\end{array}$ & $8(7.5 \%)$ \\
\hline 4 & $\begin{array}{c}\text { Failure of healthcare workers to practice simple control } \\
\text { measures(i.e. hand wash and changing gloves) }\end{array}$ & $4(3.7 \%)$ \\
\hline 5 & Noncompliance and poor adherence to therapy & $59(55.1 \%)$ \\
\hline 6 & Others & $1(0.9 \%)$ \\
\hline
\end{tabular}

Table 4: Practices/factors which contributes to resistance in hospitals.

attitude on antimicrobial stewardship was identified only among small fraction (15\%) of the participants (Table 5).

\section{Factors affecting KAP of health care professionals towards} antibiotic stewardship

Age $(\mathrm{p}=0.354)$, profession $(\mathrm{p}=0.52)$ and years of experience $(\mathrm{p}=0.125$ respectively) are not significant predictors of health care professionals towards antibiotic stewardship.

\section{Correlation between knowledge, attitude and practice}

There were no observed correlation between knowledge and attitude $(\mathrm{R}=0.117, \mathrm{p}=0.229)$, knowledge with practice $(\mathrm{R}=010 \mathrm{p}=0.921)$, and attitude with practice $(\mathrm{R}=0.98, \mathrm{p}=0.915)$ (Tables 6-8).

\section{Discussion}

A majority of the HCP in our study was males and most of them are nurses. Eighty eight percent of them were belongs to age of 20-30 and eighty percent of them have an experience of 1-4 years. Majority of well aware of the global as well as the nationwide problems of antimicrobial resistance and inappropriate antibiotics use, but at the local hospital level, the antibiotic resistance was not considered as significant problem by HCP. 


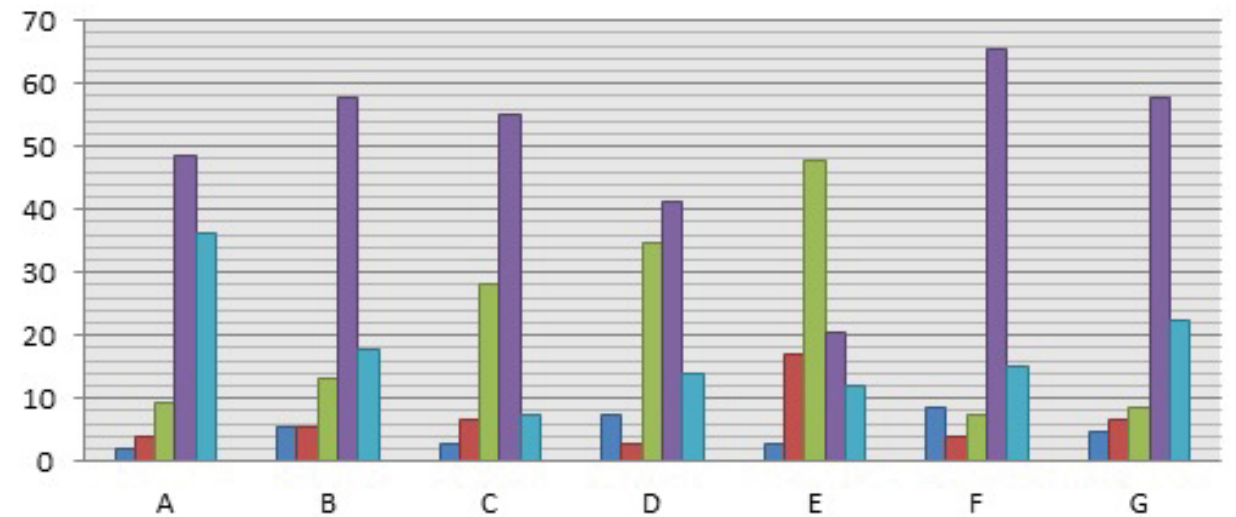

\author{
No opinion \\ - Strongly disag ree \\ $\square$ Disagree \\ agree
}

astrongly agree

\footnotetext{
A. Microbiology lab results must be provided to the treating physician.

B. If medically appropriate, IV antibiotics should be stepped down to an oral alternative after 3 days.

C. Restrictions on antibiotics impair the ability to provide good patient care.

D. More cautious use of antibiotics would decrease antimicrobial resistance.

E. Broad spectrum antibiotics should be used in place of narrow spectrum antibiotics to reduce resistance.

F. Patient rooms are cleaned according to hospital cleaning protocol.

G. This institution should provide adequate staff education regarding MDROs.
}

Figure 5: Antibiotic prescribing and other practices..

\begin{tabular}{|c|c|c|c|}
\hline \multicolumn{2}{|c|}{} & Category & Frequency (\%) \\
\hline \multirow{2}{*}{1} & \multirow{2}{*}{ Knowledge } & Good & $73(62.8 \%)$ \\
\cline { 3 - 4 } & \multirow{2}{*}{2} & Poor & $34(31.8 \%)$ \\
\hline \multirow{2}{*}{3} & \multirow{2}{*}{ Attitude } & Positive & $16(15 \%)$ \\
\cline { 3 - 4 } & \multirow{2}{*}{ Practice } & Negative & $91(85 \%)$ \\
\cline { 3 - 4 } & & Good & $78(72.9 \%)$ \\
\hline
\end{tabular}

Table 5: Level of KAP of health care professionals towards antimicrobial stewardship.

\begin{tabular}{|c|c|c|c|}
\hline \multirow{2}{*}{ Knowledge } & Knowledge & Attitude \\
\cline { 2 - 4 } & Pearson Correlation & 1 & .117 \\
\cline { 2 - 4 } & Sig. (2-tailed) & - & .229 \\
\hline \multirow{3}{*}{ Attitude } & N & 107 & 107 \\
\cline { 2 - 4 } & Pearson Correlation & 0.117 & 1 \\
\cline { 2 - 4 } & Sig. (2-tailed) & 0.229 & - \\
\cline { 2 - 4 } & $\mathrm{N}$ & 107 & 107 \\
\hline
\end{tabular}

Table 6: Correlation between knowledge and attitude.

\begin{tabular}{|c|c|c|c|}
\hline \multirow{2}{*}{ Knowledge } & Knowledge & Practice \\
\cline { 2 - 4 } & Searson Correlation & 1 & -0.010 \\
\cline { 2 - 4 } & Sig. (2-tailed) & - & 0.921 \\
\hline \multirow{3}{*}{ Practice } & N & 107 & 107 \\
\cline { 2 - 4 } & Searson Correlation & -0.010 & 1 \\
\cline { 2 - 4 } & Sig. (2-tailed) & 0.921 & - \\
\hline
\end{tabular}

Table 7: Correlation between knowledge and practice.

The level of knowledge of the respondents showed that $62.8 \%$ of HCPs had good knowledge. Majority of HCP know that inappropriate antibiotics use can lead to resistance, ineffective treatment, increased adverse effects and additional burden of medical cost to the patient. But still significant amount of healthcare professionals were not agreed with those statements. This shows lack of basic knowledge and absences of proper training regarding antibiotics use.

\begin{tabular}{|c|c|c|c|}
\hline \multicolumn{2}{|c|}{} & Attitude & Practice \\
\hline \multirow{3}{*}{ Attitude } & Pearson Correlation & 1 & -0.098 \\
\cline { 2 - 4 } & Sig. (2-tailed) & - & 0.315 \\
\cline { 2 - 4 } & $\mathrm{N}$ & 107 & 107 \\
\hline \multirow{3}{*}{ Practice } & Pearson Correlation & -0.098 & 1 \\
\cline { 2 - 4 } & Sig. (2-tailed) & 0.315 & - \\
\cline { 2 - 4 } & $\mathrm{N}$ & 107 & 107 \\
\hline
\end{tabular}

Table 8: Correlation between attitude and practice.

A significant percentage of HCP had never heard of the term AS (10.3\%) which was low when compared to study conducted in Utah which is $15 \%$ and $44.9 \%$ of HCP stated they were, not familiar with i.e., heard the term but not sure what it is. This might be due to the absence of education, basic training and promotion of antimicrobial stewardship program across the country. Familiarity of HCP with the antibiogram was very poor when compared to the same study conducted in Utah [18]. However familiarity of healthcare professionals with antibiotics resistance (81.3\%), DDD and DOT (61\%) was appreciable.

About half (50.5\%) of providers feel ASP is effective in improving patient outcomes, $(45.8 \%)$ in improving patient safety, in reducing resistance $(41.1 \%)$ and reducing healthcare costs i.e., without adversely impacting quality of care (34.6\%). There are very few HCP who feel antibiotics stewardship is not applicable for the above mentioned points.

The attitude of the study participants with regards to antibiotic use and resistance was found to be casual and lax. Thirty nine point three $(39.3 \%)$ of HCP was not believed antimicrobial are used in their hospital/facility. Forty three percent $(43 \%)$ of HCP do not feel that antimicrobials are overused in their hospital.

Regarding the use of antibiotics and antimicrobial resistance $12.1 \%$ of the participants agreed that, appropriate use of antimicrobials can lead to resistance, $39.3 \%$ of them though strongly disagreed with this statement. More than half of respondent (64.5\%) thought that new antimicrobial development will maintain with our current resistance. 
Forty three point two percent of the participants disagreed on restriction of antimicrobials use as reasonable method for controlling antibiotic use whereas $31.8 \%$ were agreed on the statement. Less than half of the respondents (44.9\%) believe that antimicrobial resistance is a serious public health issue which is comparably similar with the study done in Amara region [15].

Sixty five point four $(65.4 \%)$ of the participants were agreed and about $22.5 \%$ strongly agreed on the importance of education about antimicrobial stewardship for Healthcare professionals, which was not appreciable when compared to the study that was done in Utah [18] which is $38 \%$ and study that was done in amhara region Ethiopia [15]. Almost $910 \%$ of HCPs said they have no infectious disease specialist do not contact infectious disease specialist, which was not appreciable to one study done in Utah answered they would contact ID 1 or 2 times per month (LCH 53\%, SCH 62\%) or at least 3 times per month ( $\mathrm{LCH}$ $25 \%$, SCH $21 \%$ ) [18]. About $68.2 \%$ of the healthcare professionals used standard treatment guidelines for dealing with infectious diseases followed by clinical text books and PubMed and up-to-date. Majority of HCPs were supposed specific interventions are necessary to ensure optimal use of antibiotics to treat community-acquired pneumonia $(87.9 \%)$, urinary tract infection $(72 \%)$, skin and soft tissue infections (57\%) surgical prophylaxis $(69.2 \%)$ and for Empiric treatment of methicillin-resistant Staphylococcus aureus (86\%).

Community acquired infections are indicated as prone to increased risk of resistance (57.9\%) than nosocomial infections (42.1\%). Majority of healthcare professionals thought full recovery is a clinical state for the patient to switch from intravenous to oral antibiotics. This indicates poor level of practice in hospitals. Noncompliance and poor adherence to therapy showed as the main factor for the development of resistance of antibiotics (55.1\%) which is not appreciable when compared to the study done in amhara region which is $98 \%$, followed by intensive and prolonged antimicrobial therapy (28\%) still not comparable with the same study which is 78.4 [15].

In terms of practices related to prescribing, $48.5 \%$ agreed and $36.4 \%$ strongly agree that microbiology lab results must be provided to prescribers. Fifty five point one (55.1\%) of the respondents said restrictions on antibiotics impair their ability to provide good patient care.

Sixty two percent (62\%) of HCP agreed and ninety percent (19\%) strongly agreed IV antibiotics should be stepped down to an oral alternative after 3 days if medically appropriate. More than half (59\%) of the respondents agreed more cautious use of antibiotics would decrease antimicrobial resistance. Thirty five percent $(35 \%)$ of the HCP responded broad spectrum antibiotics should be used in place of narrow spectrum antibiotics to reduce resistance. This may be partially due to lack of knowledge and continuous training of HCP about antibiotics use and resistance [19]. Most (80\%) of respondent's agreed that patient rooms should be cleaned according to hospital cleaning protocol. Almost $68 \%$ of the HCPs agreed and $22.4 \%$ strongly agreed their institution should provide adequate staff education regarding Multidrug Resistant Organisms (MDROs).

\section{Conclusion}

Majority of HCPs showed good knowledge and practice. However most of them showed negative attitude towards it. There were no observed correlation between knowledge and attitude $(\mathrm{R}=0.117$, $\mathrm{p}=0.229)$, knowledge with practice $(\mathrm{R}=010 \mathrm{p}=0.921)$, and attitude with practice $(\mathrm{R}=0.98, \mathrm{p}=0.915)$. Accordingly, the ministry of health should develop and adopt guidelines and policies to implement and monitor antimicrobial stewardship in all government and private health institutions country wide.

\section{References}

1. https://www.fda.gov/ForConsumers/

2. Gerding DN (2001) The search for good antimicrobial stewardship. Jt Comm J Qual Improv 27: 403-404

3. Joseph J, Rodvold KA (2008) The role of carbapenems in the treatment of severe nosocomial respiratory tract infections. Expert Opin Pharmacother 9: 561-575.

4. Doron S, Davidson LE (2011) Antimicrobial Stewardship. Mayo Clin Proc 86: 1113-1123.

5. McGowan JE Jr (2005) Antimicrobial resistance in hospital organisms and its relation to antibiotic use. Rev Infect Dis $5: 1033-1048$.

6. Monroe S, Polk R (2000) Antimicrobial use and bacterial resistance. Curr Opin Microbiol 3: 496-501.

7. Yenet $W(2005)$ Base line survey on drug prescribing indicators for our patients in JUSH .south west Ethiopia. Ethiopian Health Science 15: 148-151.

8. Mekuriya T, Kebede E, Tarekegn M (2015) Antimicrobial Use-Related Problems and Predictors among Hospitalized Medical Inpatients in Southwest Ethiopia: Prospective Observational Study. Plos ONE 10: 1-9.

9. Abule T, Kedir M (2004) The pattern of antibiotic usage in surgical in-patients of a teaching hospital, northwest Ethiopia. Ethiop J Health Dev 18: 35-38.

10. Chamber HF, Brunton JS, Lazo KL (2001) Chemotherapy of microbial disease. Laurence In. Goodman and Gilman's the pharmacological basis of therapeutics (11 th edn.). McGraw Hill, Toronto, Canada pp: 1095-1108.

11. Dellit TH, Owens RC, McGowan JE Jr (2007) Infectious Diseases Society of America and the Society for Healthcare Epidemiology of America guidelines for developing an institutional program to enhance antimicrobial stewardship. Clin Infect Dis 44: 159-177.

12. Shlaes DM, Gerding DN, John JF Jr, Craig WA, Bornstein DL, et al. (1997) Society for Healthcare Epidemiology of America and Infectious Diseases Society of America Joint Committee on the Prevention of Antimicrobial Resistance: guidelines for the prevention of antimicrobial resistance in hospitals. Clin Infect Dis 25: $584-599$.

13. Siegel JD, Rhinehart E, Jackson M (2006) The Centers for Disease Control and Prevention Healthcare Infection Control Practices Advisory Committee. Management of multi-drug resistant organisms in healthcare settings.

14. Ministry of Health (1994) Master plan for the Ethiopian national drugs programme. Addis Ababa pp: 1-20.

15. Abera B, Kibret M, Mulu W (2014) Knowledge and beliefs on antimicrobia resistance among physicians and nurses in hospitals in Amhara Region, Ethiopia. BMC Pharmacol Toxicol 15: 26.

16. Barbosa TM, Levy SB (2000) The impact of antibiotic use on resistance development and persistence. Drug Resist Update 3: 303-311.

17. Murray BE, Moellering RC Jr (1978) Patterns and mechanism of antibiotic resistance. Med Clin North Am 62: 899-923.

18. Bucke W, Hersh A, Pavia A, Jones P, Caraccio J, et al. (2016) Antimicrobial Stewardship Knowledge, Attitudes and Practices among Healthcare Professionals in Utah.

19. Christian RF (2002) A strategy for the improvement of prescribing and drug use in rural facilities in Uganda, Entebe: Ministry of Health, Uganda Essential Drugs Management Programme. 